\title{
Radiotherapy for locally recurrent rectal cancer treated with surgery alone as the initial treatment
}

\author{
Hidekazu Tanaka, MD, PhD1, Takahiro Yamaguchi, MD¹, Kae Hachiya, MD', Sunaho Okada, MD', \\ Masashi Kitahara, $\mathrm{RT}^{2}$, Katsuya Matsuyama, $\mathrm{RT}^{2}$, Masayuki Matsuo, MD, PhD' \\ 'Department of Radiology, Gifu University, Gifu; \\ ${ }^{2}$ Division of Radiation Oncology, Gifu University Hospital, Gifu, Japan
}

Purpose: Although the technical developments of radiotherapy have been remarkable, there are currently few reports on the treatment results of radiotherapy for local recurrence of rectal cancer treated with surgery alone as initial treatment in this three-dimensional conformal radiotherapy era. Thus, we retrospectively evaluated the treatment results of radiotherapy for local recurrence of rectal cancer treated with surgery alone as the initial treatment.

Materials and Methods: Thirty-two patients who underwent radiotherapy were enrolled in this study. The dose per fraction was 2.0-3.5 Gy. Because the treatment schedule was variable, the biological effective dose (BED) was calculated.

Results: Local control (LC) and overall survival (OS) rates from the completion of radiotherapy were calculated. The 1-, 2-, 3-, 4-, and 5-year LC rates were 51.5\%, 24.5\%, 19.6\%, 19.6\%, and 13.1\%, respectively. LC rates were significantly higher for the high BED group $\left(\geq 75 \mathrm{~Gy}_{10}\right.$ ) than for the lower BED group $\left(<75 \mathrm{~Gy}_{10}\right)$. All patients who reported pain achieved pain relief. The duration of pain relief was significantly higher for the high BED group than for the lower BED group. The 1-, 2-, 3-, 4-, and 5-year OS rates were $82.6 \%, 56.5 \%, 45.2 \%, 38.7 \%$, and 23.2\%, respectively. There was a trend toward higher OS rates in with higher BED group compared to lower BED group.

Conclusion: For patients with unresectable locally recurrent rectal cancer treated with surgery alone, radiotherapy is effective treatment. The prescribed BED should be more than $75 \mathrm{~Gy}_{10}$, if the dose to the organ at risk is within acceptable levels.

Keywords: Rectal cancer, Radiotherapy, Locally recurrent of rectal cancer

\section{Introduction}

The local recurrence rate after curative surgery for the rectal cancer is 5\%-20\% [1-3]. The most effective treatment for local recurrence of rectal cancer is resection [4-7]. However, curative excision is possible in fewer than half of patients with recurrent rectal cancer [6-8]. Radiotherapy or chemoradiotherapy are the alternative treatments for unresectable recurrent cases [9]. Various symptoms are reported in $71 \%-89 \%$ of patients with locally recurrent rectal cancer $[8,10]$. Pain is the most common symptom, with $50 \%-64 \%$ of patients with locally recurrent rectal cancer complaining of pain $[8,10,11]$. These patients are also indicated for radiotherapy.

In recent years, several studies have revealed that preoperative or postoperative radiotherapy reduces the risk of local recurrence of the rectal cancer [3,12-14]. Radiotherapy is usually performed as a part of the initial treatment of rectal cancer. When local recurrence occurred in patients who had

Received 1 November 2016, Revised 4 January 2017, Accepted 7 February 2017.

Correspondence: Hidekazu Tanaka, MD, PhD, Department of Radiology, Gifu University, Yanagido 1-1, Gifu 501-1194, Japan. Tel: +81-58-230-6439, Fax: +81-58-230-6440, E-mail: htanaka-gif@umin.ac.jp

(c) This is an Open Access article distributed under the terms of the Creative Commons Attribution Non-Commercial License (http://creativecommons.org/ licenses/by-nc/4.0/) which permits unrestricted non-commercial use, distribution, and reproduction in any medium, provided the original work is properly cited.

www.e-roj.org 
already received radiotherapy as part of the initial treatment, the salvage radiotherapy administered was generally reirradiation $[4,6]$. Most data on the treatment results of radiotherapy for local recurrence of rectal cancer in patients treated with surgery alone as the initial treatment come from old reports $[10,11]$. In these reports, radiotherapy was performed with parallel opposed fields. Although the technical development of radiotherapy has been remarkable in recent years, there are currently no reports on the treatment results of radiotherapy for local recurrence of rectal cancer patients treated with surgery alone as the initial treatment in this era of three-dimensional conformal radiotherapy (3D-CRT).

Total mesorectal excision or tumor-specific mesorectal excision with lateral node dissection are the most selected operative procedures in Japan, and radiotherapy is often omitted as part of the initial treatment of rectal cancer. Therefore, local recurrence of rectal cancer treated with surgery alone as the initial treatment is relatively common in Japan. In the current study, we retrospectively evaluated the treatment results of radiotherapy for local recurrence of rectal cancer treated with surgery alone as the initial treatment.

\section{Materials and Methods}

This study was approved by the Institutional Review Board. All patients provided written informed consent before undergoing radiotherapy. Thirty-two patients who underwent radiotherapy in our hospital between April 2005 and October 2014 were enrolled in this study. Patients who had received radiotherapy as part of their initial treatment were excluded from the study. Most diagnoses of recurrence were made by the observation of increasing size of a local region and the elevation of tumor markers. Only 4 patients were confirmed with recurrence by biopsy. The median age was 67 years (range, 41 to 81 years). The median time from primary surgery to any failure (including local recurrence) was 24 months (range, 3 to 99 months). The median time from primary surgery to local recurrence was 30 months (range, 4 to 99 months). At the time of radiation for local recurrence, 14 patients had other regional or distant metastasis. At the time of radiation therapy for local recurrence, 14 patients had other regional or distant metastases, including 2, 10, and 2 cases of regional node metastasis, distant metastasis, and regional and distant metastasis, respectively. The remaining 18 patients had local recurrences only. Seventeen patients (53.1\%) with local recurrence were considered medically inoperable owing to factors such as the extent of infiltration to the adjacent
Table 1. Patients' characteristics $(n=32)$

\begin{tabular}{|c|c|}
\hline Characteristic & Value \\
\hline Age (yr) & $67(41-81)$ \\
\hline \multicolumn{2}{|l|}{ Sex } \\
\hline Male & 18 \\
\hline Female & 14 \\
\hline \multicolumn{2}{|l|}{ ECOG performance status } \\
\hline 0 & 17 \\
\hline 1 & 14 \\
\hline 2 & 1 \\
\hline \multicolumn{2}{|l|}{ Postoperative stage } \\
\hline I & 3 \\
\hline$\|$ & 6 \\
\hline IIIA & 14 \\
\hline IIIB & 6 \\
\hline IV (liver metastasis) & 3 \\
\hline Time from primary surgery to any failure (mo) & $24(3-99)$ \\
\hline $\begin{array}{l}\text { Time from primary surgery to local recurrence } \\
\text { (mo) }\end{array}$ & $30(4-99)$ \\
\hline \multicolumn{2}{|l|}{ Recurrent site } \\
\hline Axial & 11 \\
\hline Anterior & 3 \\
\hline Posterior & 15 \\
\hline Lateral & 3 \\
\hline \multicolumn{2}{|l|}{ Metastasis } \\
\hline Regional & 2 \\
\hline Regional and distant & 2 \\
\hline Distant & 10 \\
\hline None & 18 \\
\hline Tumor size $(\mathrm{mm})$ & $39(12-150)$ \\
\hline CEA (ng/mL) & $12.7(1.5-774)$ \\
\hline \multicolumn{2}{|l|}{ Pain } \\
\hline Yes & 22 \\
\hline No & 10 \\
\hline $\operatorname{BED}\left(\mathrm{Gy}_{10}\right)$ & $72.0(48.0-95.2)$ \\
\hline$\geq 75$ & 7 \\
\hline$<75$ & 25 \\
\hline \multicolumn{2}{|l|}{ Concurrent chemotherapy } \\
\hline Yes & 20 \\
\hline No & 12 \\
\hline \multicolumn{2}{|l|}{ Adjuvant chemotherapy after RT } \\
\hline Yes & 24 \\
\hline No & 8 \\
\hline Follow-up period (mo) & $16(4-108)$ \\
\hline
\end{tabular}

Values are presented as median (range).

ECOG, Eastern Cooperative Oncology Group; CEA, carcinoembryonic antigen; BED, biological effective dose with $\alpha / \beta=10 \mathrm{~Gy}$; RT, radiotherapy.

organs; 12 patients (37.5\%) were not suited for salvage surgery because of the presence of distant metastases, while 3 patients (9.4\%) refused salvage surgery. Twenty-two patients 
(68.8\%) reported pain due to the local recurrence. The local recurrence site was classified into 4 groups, as presented by Yeo and Paty [15]: (1) axial, which can be subdivided into anastomotic, mesorectal or perirectal soft tissue, or perineum; (2) anterior, involving the genitourinary tract including the bladder, vagina, uterus, seminal vesicles, and prostate; (3) posterior, involving the sacrum and presacral fascia; and (4) lateral, involving the soft tissues of the pelvic sidewall and lateral bony pelvis. Patient characteristics are shown in Table 1. The median follow-up period after the completion of radiotherapy was 16 months (range, 4 to 108 months).

The 3D-CRT was performed with 3-6 fields with a Clinac 21 (Varian Medical Systems, Palo Alto, CA, USA). The multileaf collimator leaf width was $5 \mathrm{~mm}$. In principal, the irradiated field was limited to recurrent tumors. Two patients with regional lymph node metastasis received irradiation in both the local and regional recurrence sites. The planning target volume (PTV) margins were 5-10 $\mathrm{mm}$. The dose fractionation schedules are shown in Table 2. In most cases, the dose per fraction was 2.0-3.5 Gy. Only two patients were irradiated with 7 Gy per fraction as a boost irradiation. A high dose was irradiated in patients in whom irradiation to the surrounding organs-atrisk (OARs), such as the small bowel, could be avoided. Patients with posterior recurrence or with colostomy tended to be irradiated at a relatively high dose. Because the treatment schedule was variable, the biological effective dose (BED) was calculated. Local control (LC) and overall survival (OS) rates from the completion of radiotherapy were calculated using the Kaplan-Meier method and group comparisons were made using the log-rank test. Group comparisons included time form primary surgery to recurrence ( $\leq 6$ months vs. $>6$ months), site of recurrence, presence or absence of distant metastasis, serum carcinoembryonic antigen $(\geq 5.0 \mathrm{ng} / \mathrm{mL}$ vs. $<5.0 \mathrm{ng} /$ $\mathrm{mL}$ ), recurrent tumor size ( $\geq 4 \mathrm{~cm}$ vs. $<4 \mathrm{~cm}$ ), BED ( $\geq 75 \mathrm{~Gy} y_{10}$ vs. $<75 \mathrm{~Gy}_{10}$ ), presence or absence of concurrent chemotherapy, and presence or absence of adjuvant chemotherapy after radiotherapy. A p-value less than 0.05 was considered to indicate a statistically significant difference.

\section{Results}

\section{Local control}

During the follow-up period, local recurrence was observed in 21 patients. Most local recurrences occurred in the irradiated field. Only 2 cases of recurrence at the marginal irradiation region were observed. The 1-, 2-, 3-, 4-, and 5-year LC rates were $51.5 \%, 24.5 \%, 19.6 \%, 19.6 \%$, and $13.1 \%$, respectively
Table 2. Dose fractionation schedule $(n=32)$

\begin{tabular}{lcc}
\hline \multicolumn{1}{c}{ Dose fractionation } & BED $\left(\mathrm{Gy}_{10}\right)$ & No. of patients \\
\hline $48 \mathrm{~Gy}$ in $20 \mathrm{fx}+21 \mathrm{~Gy}$ in $3 \mathrm{fx}$ & 95.2 & 2 \\
$74.25 \mathrm{~Gy}$ in $33 \mathrm{fx}$ & 91.0 & 1 \\
$66 \mathrm{~Gy}$ in $33 \mathrm{fx}$ & 79.2 & 2 \\
$64 \mathrm{~Gy}$ in $32 \mathrm{fx}$ & 76.8 & 2 \\
$60 \mathrm{~Gy}$ in $30 \mathrm{fx}$ & 72.0 & 10 \\
$40.8 \mathrm{~Gy}$ in $17 \mathrm{fx}+15 \mathrm{~Gy}$ in $6 \mathrm{fx}$ & 69.3 & 2 \\
$55 \mathrm{~Gy}$ in $22 \mathrm{fx}$ & 68.8 & 2 \\
$54 \mathrm{~Gy}$ in $27 \mathrm{fx}$ & 64.8 & 2 \\
$50 \mathrm{~Gy}$ in $20 \mathrm{fx}$ & 60.0 & 1 \\
$48 \mathrm{~Gy}$ in $16 \mathrm{fx}$ & 62.4 & 1 \\
$50 \mathrm{~Gy}$ in $25 \mathrm{fx}$ & 60.0 & 3 \\
$48 \mathrm{~Gy}$ in $20 \mathrm{fx}$ & 59.5 & 2 \\
$39 \mathrm{~Gy}$ in $13 \mathrm{fx}$ & 50.7 & 1 \\
$40 \mathrm{~Gy}$ in $20 \mathrm{fx}$ & 48.0 & 1 \\
\hline
\end{tabular}

$\mathrm{BED}$, biological effective dose with $\alpha / \beta=10 \mathrm{~Gy}$; fx, fractions.

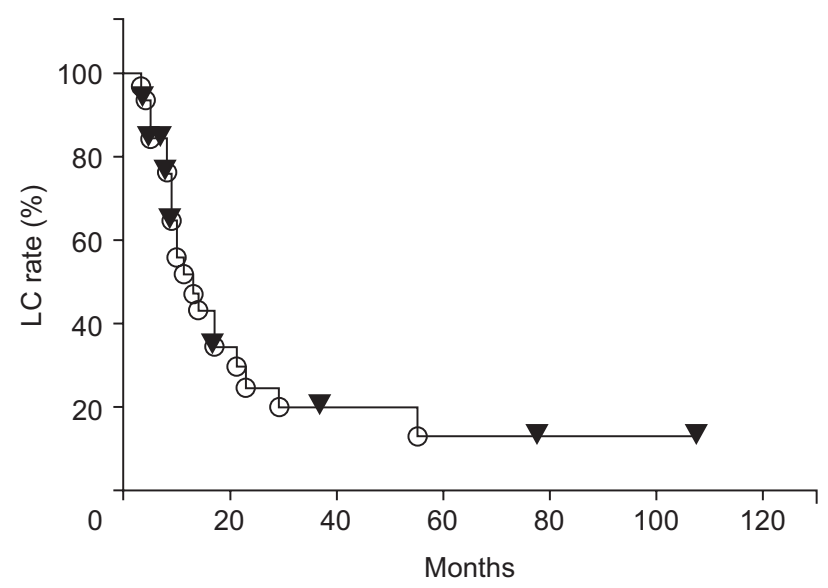

Fig. 1. Local control (LC) rate curves after radiotherapy.

(Fig. 1). The $L C$ rates were significantly higher for the high BED group than for the lower BED group ( $p=0.0243)$ (Fig. 2). There were no significant differences in other group comparisons (Table 3).

All patients who reported pain achieved pain relief. The median duration of pain relief was 10 months (range, 3 to 108 months). The duration of pain relief was significantly higher for the high BED group than for the lower BED group ( $p=$ 0.0311).

\section{Overall survival}

Fifteen patients died during the follow-up periods. All deaths were due to the rectal cancer. The exact causes of deaths were pulmonary metastases, hepatic metastases, peritonitis carcinomatosa, and unknown in 8, 4, 2, and 1 case, 
Table 3. Summary of univariate analysis of $L C$ rate

\begin{tabular}{|c|c|c|}
\hline Factor & $1-y r$ LC rate $(\%)$ & p-value \\
\hline $\begin{array}{l}\text { Time from primary surgery to } \\
\text { recurrence (mo) }\end{array}$ & & 0.1142 \\
\hline$\leq 6$ & 37.5 & \\
\hline$>6$ & 53.2 & \\
\hline Site of recurrence & & 0.2309 \\
\hline Axial & 51.9 & \\
\hline Anterior & 33.3 & \\
\hline Posterior & 43.2 & \\
\hline Lateral & 100 & \\
\hline Presence of distant metastasis & & 0.4672 \\
\hline No & 50.7 & \\
\hline Yes & 55.6 & \\
\hline Serum CEA (ng/mL) & & 0.0988 \\
\hline$\geq 5.0$ & 55.6 & \\
\hline$<5.0$ & 25.0 & \\
\hline Recurrence tumor size (cm) & & 0.9066 \\
\hline$<4.0$ & 56.9 & \\
\hline$\geq 4.0$ & 45.8 & \\
\hline BED $\left(\mathrm{Gy}_{10}\right)$ & & 0.0243 \\
\hline$\geq 75$ & 83.3 & \\
\hline$<75$ & 41.9 & \\
\hline Concurrent chemotherapy & & 0.8903 \\
\hline Yes & 49.5 & \\
\hline No & 53.0 & \\
\hline Adjuvant chemotherapy & & 0.5419 \\
\hline Yes & 53.9 & \\
\hline No & 43.8 & \\
\hline
\end{tabular}

LC, local control; CEA, carcinoembryonic antigen; BED, biological effective dose with $\alpha / \beta=10 \mathrm{~Gy}$.

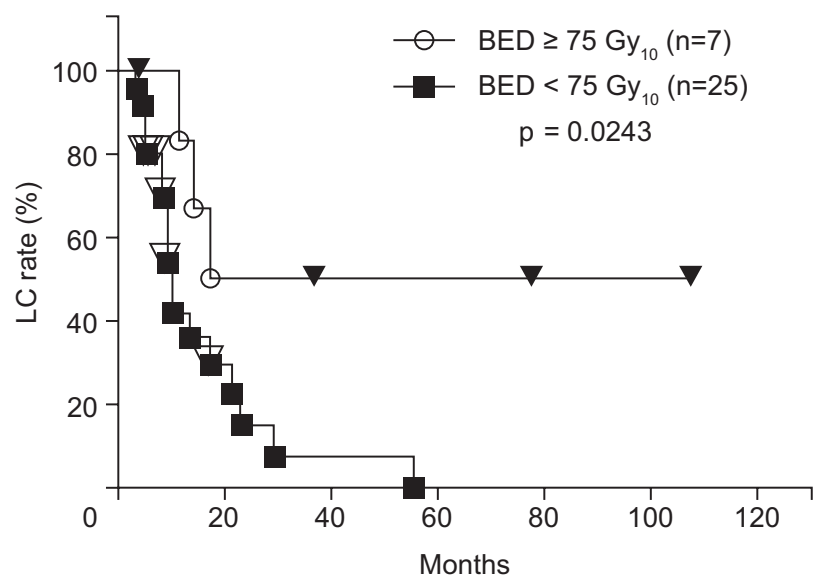

Fig. 2. Local control (LC) rate curves after radiotherapy. $L C$ rates were significantly higher in the high biological effective dose (BED) group than in the low BED group.
Table 4. Summary of univariate analysis of OS rate

\begin{tabular}{|c|c|c|}
\hline Factor & $1-y r$ OS rate $(\%)$ & $p$-value \\
\hline $\begin{array}{l}\text { Time from primary surgery to } \\
\text { recurrence (mo) }\end{array}$ & & 0.0003 \\
\hline$\leq 6$ & 50.0 & \\
\hline$>6$ & 87.9 & \\
\hline Site of recurrence & & 0.2309 \\
\hline Axial & 77.1 & \\
\hline Anterior & 66.7 & \\
\hline Posterior & 85.6 & \\
\hline Lateral & 100 & \\
\hline Presence of distant metastasis & & 0.049 \\
\hline No & 89.2 & \\
\hline Yes & 71.3 & \\
\hline Serum CEA (ng/mL) & & 0.9336 \\
\hline$\geq 5.0$ & 83.7 & \\
\hline$<5.0$ & 75.0 & \\
\hline Recurrence tumor size $(\mathrm{cm})$ & & 0.3298 \\
\hline$<4.0$ & 79.3 & \\
\hline$\geq 4.0$ & 86.2 & \\
\hline BED $\left(\mathrm{Gy}_{10}\right)$ & & 0.0730 \\
\hline$\geq 75$ & 100 & \\
\hline$<75$ & 77.9 & \\
\hline Concurrent chemotherapy & & 0.6065 \\
\hline Yes & 84.4 & \\
\hline No & 80.0 & \\
\hline Adjuvant chemotherapy & & 0.8559 \\
\hline Yes & 82.6 & \\
\hline No & 75.0 & \\
\hline
\end{tabular}

OS, overall survival; $C E A$, carcinoembryonic antigen; $B E D$, biological effective dose with $\alpha / \beta=10 \mathrm{~Gy}$.

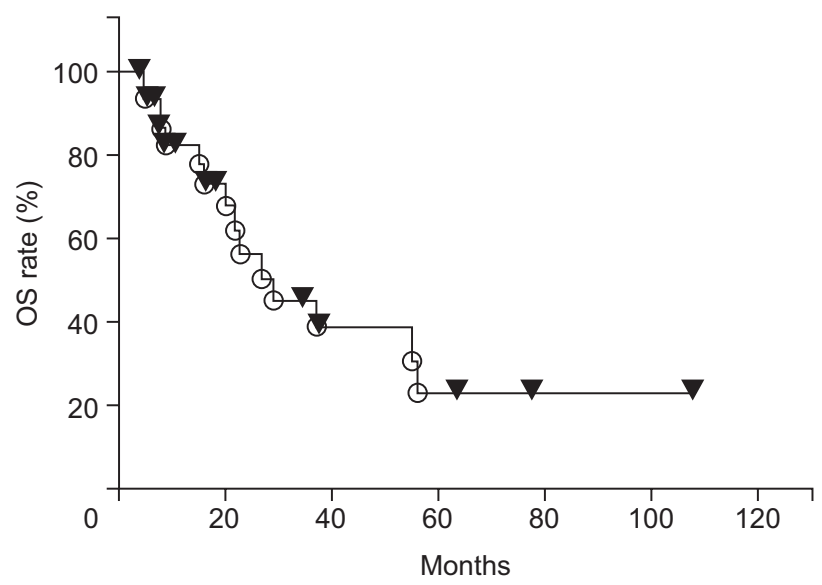

Fig. 3. Overall survival (OS) curves after radiotherapy. 
respectively.

The 1-, 2-, 3-, 4-, and 5-year OS rates were 82.6\%, 56.5\%, $45.2 \%, 38.7 \%$, and 23.2\%, respectively (Fig. 3). There was a trend toward higher OS rates in patients in the higher BED group compared to those in the lower BED group ( $p=0.0730)$ (Fig. 4). The OS rates were significantly lower for the patients with recurrence before 6 months from primary surgery than for the patients with recurrence after 6 months from primary surgery $(p=0.0003)$. In the patients without distant metastases (local recurrence \pm regional node metastasis only), the 1-, 2-, 3-, 4-, 5-year OS rates were 89.2\%, 64.0\%, 64.0\%, $53.3 \%$, and $32.0 \%$, respectively. In the patients with distant metastases, the 1-, 2-, and 3-year OS rates were 71.3\%, 42.8\%, and $14.3 \%$, respectively. The $0 \mathrm{~S}$ rates were significantly higher for the patients without distant metastases ( $p=0.0490)$ (Fig. 5). There were no significant differences in other group comparisons (Table 4).

\section{Discussion and Conclusion}

In recent years, the opportunities to irradiate rectal cancer preoperatively or postoperatively have increased. In Japan, total mesorectal excision or tumor-specific mesorectal excision with lateral node dissection are the standard operative procedures, and radiotherapy is often omitted as part of the initial treatment for rectal cancer. Therefore, local recurrence of rectal cancer treated with surgery alone as the initial treatment is not so rare in Japan. Current knowledge

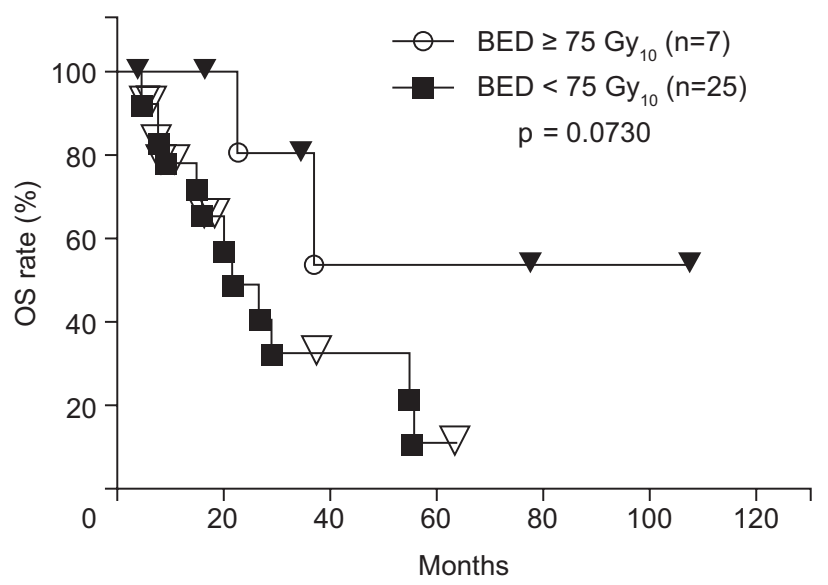

Fig. 4. Overall survival (OS) curves after radiotherapy. There was a trend toward higher OS rates in patients in the higher biological effective dose (BED) group compared to those in the lower BED group. regarding the treatment results of radiotherapy for local recurrence of rectal cancer treated with surgery alone as the initial treatment were based on previous reports. Ciatt Pacini [11] reported that the 3-year and 5-year OS rates of patients who underwent irradiation for recurrent rectal cancer were $5 \%$ and $3 \%$, respectively. Knol et al. [10] reported that the 2and 5-year OS rates of patients who underwent irradiation for recurrent rectal cancer without distant metastasis were 33\% and $12 \%$, respectively. In our study, the $2-, 3-$, and 5-year OS rates were $56.5 \%, 45.2 \%$, and $23.2 \%$, respectively, which are better than the findings of these previous reports. The $L C$ rates were significantly higher for the high BED group than for the lower BED group. There was a trend toward higher OS rates in the higher BED group compared to the lower BED group. A prescribed $\mathrm{BED}$ of $\geq 75 \mathrm{~Gy}_{10}$ might be desirable if the dose to the OAR is within acceptable levels.

The radiation therapy techniques are rapidly developing. Image-guided radiation therapy (IGRT) allows the reduction of set-up errors, resulting in reduced PTV margin. Therefore, the irradiated dose to the adjacent OAR might be lower. Consequently, physicians might prescribe higher doses more easily, as compared to in the era before the application of IGRT. Moreover, intensity-modulated radiation therapy is another promising method for reducing the dose to the adjacent OAR. A reduced dose to the OARs might lead to a high dose prescription to the target volume.

Only 2 patients received salvage surgery after radiotherapy. One patient experienced in-field recurrence at 23 months after

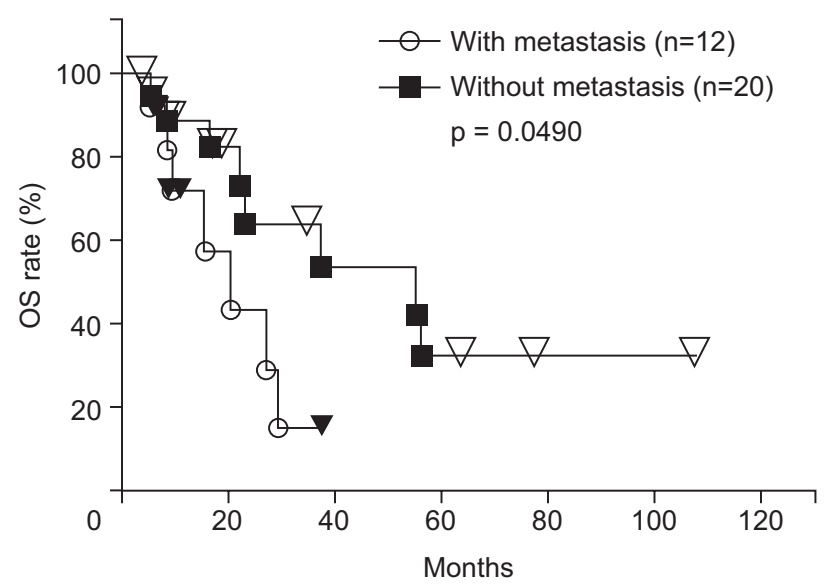

Fig. 5. Overall survival (OS) curves after radiotherapy. The OS rates were significantly higher for the patients without distant metastases than for those with metastases. 
salvage irradiation with $40.0 \mathrm{~Gy}$ for a first recurrence at the axial site. The patient refused surgery at the detection of first recurrence. At the detection of a second recurrence, the patient accepted the proposal of salvage surgery. Unfortunately, liver metastasis was found 5 months after the salvage surgery and the patient died from cancer 56 months after the salvage radiotherapy, despite also receiving chemotherapy. The other patient experienced in-field recurrence at 17 months after salvage irradiation with $60.8 \mathrm{~Gy}$ for a first recurrence at the posterior site. The second recurrent tumor was relatively small and did not seem to invade the sacrum, likely due to use of salvage radiation therapy. Subsequently, salvage operation was performed. At the latest follow-up, this patient was alive without recurrence. These findings suggested that, although the number of patients who received salvage surgery was very small in this study, long-term survival (64 months from salvage radiotherapy) was seen in one patients. Even if the recurrent tumor dose not disappear by salvage radiotherapy, the possibility of resection might be elevated due to reduction of the tumor by the radiotherapy.

Various symptoms are reported in $71 \%-89 \%$ of patients with locally recurrent rectal cancer $[8,10]$. Pain is the most common symptom, and 50\%-64\% of patients with locally recurrent rectal cancer complain of pain $[8,10,11]$. High pain relief rates of 56\%-100\% have been reported with radiotherapy [11,16-18]. In our study, the rate of pain relief was $100 \%$, and the median duration of pain relief was 10 months. The duration of pain relief was significantly higher for the high BED group than for the lower BED group. Therefore, the prescribed BED should be more than $75 \mathrm{~Gy}_{10}$, even for patients receiving palliative therapy.

In this study, most local recurrences occurred in the irradiated field. Only 2 cases of recurrence at the marginal irradiation region were observed. Therefore, the reason of recurrence might be the use of an insufficiently high irradiation dose rather than deficiency of the irradiation margin. The dose that can be irradiated for pelvic tumors is limited. Charged particle therapy is one potential solution for addressing the insufficient irradiated dose in such cases. Especially, heavy ion therapy, which involves high linear energy transfer beams, has been shown to result in a better biological effect than photon or proton beam therapy. Yamada et al. [19] reported that the 5-year LC and OS rates were $94 \%$ and $40 \%$, respectively. Although the results of charged particle therapy are excellent, it is costly and available only in a limited number of facilities. If the problems related to costs and facilities can be solved in the future, charged particle therapy may represent a highly promising option.

In conclusion, for patients with unresectable locally recurrent rectal cancer treated with surgery alone, radiotherapy is effective treatment. A prescribed BED of $\geq 75 \mathrm{~Gy}_{10}$ might be desirable if the dose to the OAR is within acceptable levels.

\section{Conflict of Interest}

No potential conflict of interest relevant to this article was reported.

\section{References}

1. Davies $M$, Harris $D$, Hirst $G$, et al. Local recurrence after abdomino-perineal resection. Colorectal Dis 2009;11:39-43.

2. Heriot $A G$, Byrne $C M$, Lee $P$, et al. Extended radical resection: the choice for locally recurrent rectal cancer. Dis Colon Rectum 2008:51:284-91.

3. Colorectal Cancer Collaborative Group. Adjuvant radiotherapy for rectal cancer: a systematic overview of 8,507 patients from 22 randomised trials. Lancet 2001;358:1291-304.

4. Mohiuddin M, Marks GM, Lingareddy V, Marks J. Curative surgical resection following reirradiation for recurrent rectal cancer. Int J Radiat Oncol Biol Phys 1997;39:643-9.

5. Wanebo HJ, Antoniuk P, Koness RJ, et al. Pelvic resection of recurrent rectal cancer: technical considerations and outcomes. Dis Colon Rectum 1999;42:1438-48.

6. Das P, Delclos ME, Skibber JM, et al. Hyperfractionated accelerated radiotherapy for rectal cancer in patients with prior pelvic irradiation. Int J Radiat Oncol Biol Phys 2010;77:60-5.

7. Bosman SJ, Holman FA, Nieuwenhuijzen GA, Martijn $H_{\text {, }}$ Creemers GJ, Rutten HJ. Feasibility of reirradiation in the treatment of locally recurrent rectal cancer. Br J Surg 2014;101:1280-9.

8. Tanis PJ, Doeksen A, van Lanschot JJ. Intentionally curative treatment of locally recurrent rectal cancer: a systematic review. Can J Surg 2013;56:135-44.

9. Beyond TME Collaborative. Consensus statement on the multidisciplinary management of patients with recurrent and primary rectal cancer beyond total mesorectal excision planes. Br J Surg 2013;100:1009-14.

10. Knol HP, Hanssens PE, Rutten HJ, Wiggers T. Effect of radiation therapy alone or in combination with surgery and/ or chemotherapy on tumor and symptom control of recurrent rectal cancer. Strahlenther Onkol 1997;173:43-9.

11. Ciatto S, Pacini P. Radiation therapy of recurrences of carcinoma of the rectum and sigmoid after surgery. Acta Radiol Oncol 1982;21:105-9. 
12. Bosset JF, Collette L, Calais G, et al. Chemotherapy with preoperative radiotherapy in rectal cancer. $\mathrm{N}$ Engl J Med 2006;355:1114-23.

13. Gerard JP, Conroy T, Bonnetain F, et al. Preoperative radiotherapy with or without concurrent fluorouracil and leucovorin in T3-4 rectal cancers: results of FFCD 9203. J Clin Oncol 2006;24:4620-5.

14. Krook JE, Moertel CG, Gunderson LL, et al. Effective surgical adjuvant therapy for high-risk rectal carcinoma. N Engl J Med 1991;324:709-15.

15. Yeo HL, Paty PB. Management of recurrent rectal cancer: practical insights in planning and surgical intervention. J Surg Oncol 2014;109:47-52.

16. O'Connell MJ, Childs DS, Moertel CG, et al. A prospective controlled evaluation of combined pelvic radiotherapy and methanol extraction residue of BCG (MER) for locally unresectable or recurrent rectal carcinoma. Int J Radiat Oncol Biol Phys 1982;8:1115-9.

17. Frykholm GJ, Pahlman L, Glimelius B. Treatment of local recurrences of rectal carcinoma. Radiother Oncol 1995;34:185-94.

18. Cameron MG, Kersten C, Vistad I, Fossa S, Guren MG. Palliative pelvic radiotherapy of symptomatic incurable rectal cancer: a systematic review. Acta Oncol 2014;53:164-73.

19. Yamada $S$, Shinoto $M$, Shigeo $Y$, et al. Current status and perspective of heavy ion beam therapy for patients with pelvic recurrence after primarily resected rectal cancer. Gan To Kagaku Ryoho 2009;36:1263-6. 